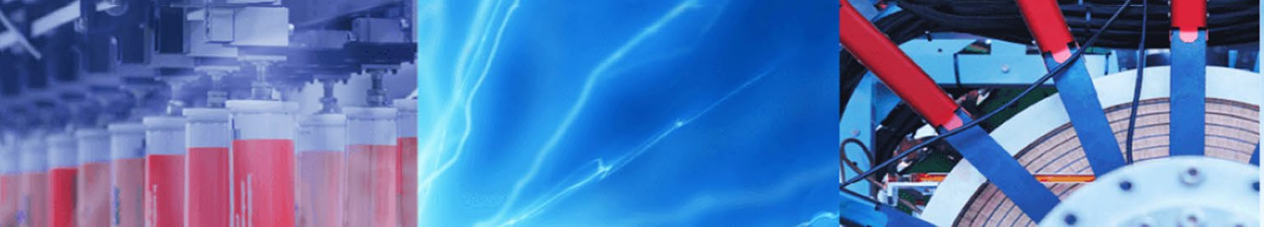

Research Article

\title{
Investigating the high-temperature performance and activation energy of carbon black-modified asphalt binder
}

\author{
Mohammad Ali Notani ${ }^{1}$ - Ali Arabzadeh ${ }^{2} \cdot$ Sajjad Satvati $^{2} \cdot$ Mahmood Tarighati Tabesh $^{1} \cdot$ Navid Ghafari Hashjin $^{3}$. \\ Shahriar Estakhri ${ }^{3}$. Masoud Alizadeh ${ }^{4}$
}

Received: 19 September 2019 / Accepted: 22 January 2020 / Published online: 30 January 2020

(c) Springer Nature Switzerland AG 2020

\begin{abstract}
This study set out to evaluate the influence of carbon black (CB) on the rheological properties and activation energy of asphalt binder at high service temperatures. The rheological performance of virgin and modified asphalt binders are investigated using three evaluation approaches: (1) Superpave specification parameter $(G * / \sin \delta)$, (2) multiple stress creep recovery (MSCR) test, and (3) interaction model for computing zero shear viscosity (ZSV). Moreover, the Arrhenius model was used to quantify the activation energy $\left(E_{f}\right)$ of virgin and CB-modified asphalt binders. The result of this study reveals that modifying asphalt binder with up to $10 \%$ of $C B$, by weight of the total asphalt binder, enhances the elastic behavior $(R \%)$ and decreases the non-recoverable creep compliance $\left(J_{n r}\right)$ of asphalt binder at high temperatures. Moreover, according to this study, the ZSV index can describe successfully rutting resistance of asphalt binder when compared with MSCR and Superpave rutting specification parameter. Besides, it was indicated that CB-modified asphalt binder has a high fluid resistance as more thermal energy was required for overcoming intermolecular force between molecules.
\end{abstract}

Keywords Rheology · Asphalt binder · Modification · Carbon black · Rutting resistance · Activation energy · Arrhenius model

\section{Introduction}

Dating back to many years ago, asphalt binder is still used for producing asphalt concrete, and it has been known as the most common construction material for paving the roadways [1]. However, researchers are still continuing to develop higher-quality asphalt binders resulting in more sustainable and resilient flexible pavements [2-5]. The overall performance of asphalt mixture is highly dependent upon the asphalt binder's intrinsic properties [6, 7]. Whereby, a high-quality asphalt binder can prevent or delay the environmental-and-traffic-related distresses in flexible pavements and hence increase the longevity of these pavements. Asphalt pavement distresses, i.e., rutting and cracking, occur before the time that flexible pavements reach their service lives $[8,9]$. These distresses result in huge amounts of money spent on rehabilitation or even reconstruction of flexible pavements; hence, it would be wiser to prevent such expenditures when it is possible to develop more sustainable and more resilient asphalt binders $[10,11]$.

High service temperature, high traffic load, asphalt mixture mechanical properties, and pavement structure are the main reasons collectively resulting in the occurrence of permanent deformation in the surface of flexible pavements [12]. Such factors contribute to cause permanent

$\triangle$ Mohammad Ali Notani, mnotani@purdue.edu; Ali Arabzadeh, arab@iastate.edu; Sajjad Satvati, ssatvati@iastate.edu; Mahmood Tarighati Tabesh, mtarigha@purdue.edu; Navid Ghafari Hashjin, navid.ghafari@aut.ac.ir; Shahriar Estakhri, shahriarestakhri@aut.ac.ir; Masoud Alizadeh, msd.alizadehv@gmail.com | ${ }^{1}$ Lyles School of Civil Engineering, Purdue University, West Lafayette, IN 47907-2051, USA. ²Civil, Construction and Environmental Engineering (CCEE), lowa State University, Ames, IA, USA. ${ }^{3}$ Department of Civil and Environmental Engineering (CEE), Amirkabir University of Technology, Tehran, Iran. ${ }^{4}$ Islamic Azad University, Tehran, Iran. 
deformation/depression on wheel paths resulting in a poor serviceability $[13,14]$. Although unrecoverable deformations in pavement layers and subgrade can collectively result in the occurrence of rutting on the pavement surface, the main reason for the occurrence of rutting is the nonlinear plastic deformation of asphalt concrete layer $[15,16]$. Sousa et al. [17] concluded that the rutting in the asphalt mixture layer could be caused by both densification and plastic deformation due to traffic loading. According to Sousa et al. [17], the plastic deformation occurs with no volume change in the asphalt mixture in both loading area and upheaval zone. The viscoelastic behavior of asphalt mixture is only because of the presence of the asphalt binder. The aggregate skeleton has no contribution to the viscoelastic behavior, although the aggregate carries the majority of traffic loading. Therefore, the rheological properties of asphalt binder affect the plastic flow in the asphalt mixture [18]. Eisemnmann and Hilmer [19] presented that the rutting, due to densification, is attributed to shear deformation without volume change. Enhancing the shear resistance of asphalt binder, through modification, would be an efficient method to mitigate this type of distress, given that there are no issues related to the mix design of asphalt concrete. For instance, numerous polymer-based modifiers can enhance the shear resistance of asphalt binder $[11,20,21]$. Although these polymer-based modifiers are added in small amounts, they can double the price of modified asphalt binder [22, 23]. To make the polymer modification method more economically viable, it is possible to use waste polymers [24, 25], or carbon-based by-products such as carbon black $(\mathrm{CB})$ that is obtained from the incomplete combustion of heavy petroleum products [26]. CB not only is a good candidate for improving the rutting resistance, but also it imparts anti-aging properties to asphalt binder [27-29] and improves the physical and rheological behavior of asphalt binder [30, 31].

Furthermore, $C B$ has a physical and chemical affinity with asphaltene and maltene that are the constituents of asphalt binder [32-34]. It has been proved that carbon atoms in the graphite-like sheets presented in the carbon black fortify carbonyl functional group $(C=O)$ and $C-O$ bonds of the asphalt binder [35]. It has been also claimed that the lighter portion of asphalt binder is absorbed by carbon black resulting a higher viscosity asphalt binder $[36,37]$. In another way, CB incorporated in crumb rubber can transform into asphalt binder by absorbing paraffin and maltenes of asphalt binder [38]. This superior performance can be attributed to the particular surface property of $C B$ having various functional groups that facilitate the interaction with hydrocarbon fluids like asphalt binder [32]. This feature may alter the bond strength between asphalt binder's hydrocarbon chains. There has been increasing interest in the use of $C B$ in asphalt mixture, because this material can be used as a conductive additive to enhance the anti-aging and thermal conductivity of asphalt mixtures [35].

In addition to experimental approaches, there has been many numerical methods investigating the permanent deformation in asphalt concrete resulting in the development of specification parameters capable of measuring as well as accounting for the rutting resistance of virgin and modified asphalt binders [39-43]. These numerical research efforts can help identify the influence of $C B$ on changing the rheological behavior of asphalt binder. Besides, such a numerical identification approach facilitates the investigation of the effectiveness of $C B$ on the high-temperature performance of asphalt binder. Moreover, CB particles interact with the light portion of asphalt binder that may change the intermolecular force due to the interaction of $C B$ particles with maltene phase of asphalt binder. This is important, because the modified asphalt binder should be fluid enough for mixing and compaction purpose. Therefore, the required activation energy, the amount of thermal energy to overcome an energy barrier of asphalt binder's atoms and molecules to move to an adjacent vacant place, is still unclear for such modified asphalt binders. Although a few studies are dedicated to the mechanical properties of $C B$, the influence of $\mathrm{CB}$ on the rheological, permanent deformation resistance, and activation energy of asphalt binder is still a matter of concern and dispute.

This study investigates the effect of carbon black on the rheological and rutting performance as well as the activation energy of asphalt binder. For this purpose, the objective of this study is, first, to characterize the viscoelastic behavior of CB-modified asphalt binder through using different approaches: (1) measuring the Superpave specification parameter, (2) calculating the average recovery percentage, (3) calculating the non-recoverable creep compliance, and (4) calculating the zero shear viscosity (ZSV). Second, evaluate the effect of CB on asphalt binder's activation energy based on Arrhenius model.

\section{Material and test methods}

\subsection{Materials and modification procedure}

In this study, a virgin (neat) asphalt binder with an $85 / 100$ penetration grade (with a performance grade of 58-22), obtained from Akam Binder Inc., was used as a base binder, and then it was modified by CB N375 obtained from Pars Carbon Black Company, Tehran, Iran. Table 1 presents the physical properties of CB used in this study. Also, Fig. 1 
Table 1 Physical properties of Carbon Black

\begin{tabular}{lrl}
\hline Properties & \multicolumn{1}{l}{ Value } & \multicolumn{1}{l}{ Standard } \\
\hline lodine adsorption number $(\mathrm{mg} / \mathrm{g})$ & $95-85$ & ASTM D1510 \\
Absorption number $\left(\mathrm{cm}^{3} / 100 \mathrm{~g}\right)$ & $119-109$ & ASTM D2414 \\
Pelleted-pour density $(\mathrm{g} / \mathrm{L})$ & $365-25$ & ASTM D1513 \\
\hline
\end{tabular}

shows the flowchart of the modification procedure and executed tests.

The $C B$ was added to virgin asphalt binder at variable rates of $5 \%, 10 \%$, and $15 \%$ by weight of total asphalt binder. As shown in Fig. 1, virgin asphalt binder and CB were blended using medium-shear radial flow mixer at a 350 revolution per minute (RPM) for $20 \mathrm{~min}$ at $163^{\circ} \mathrm{C}$. For simplicity, the virgin binder and the three-modified asphalt binders were coded as VB, CB 5\%, CB 10\%, and CB15\%.

\subsection{Test methods}

In 1993, the Strategic Highway Research Program (SHRP) introduced the dynamic shear rheometer (DSR) apparatus to characterize the rheological and viscoelastic behavior of asphalt binder. This characterization method has been inspired by the concept of torsional flow between parallel plates applying oscillatory and rotationally stresses/strains over a range of temperatures and loading frequencies [44]. The DSR applies rotational and oscillating stresses/strains to a slim film of binder located between two plates of DSR apparatus to obtain the viscoelastic parameters of asphalt binder at different temperatures.

As the foremost step, Anderson [45] introduced $G^{*} / \sin \delta$ to characterize the rutting resistance of asphalt binder that is calculated based on the two main viscoelastic parameters: complex shear modulus $\left(G^{*}\right)$ and phase angle $(\delta)$. It is supposed that permanent deformation is caused by the total dissipated energy relating this parameter to rutting behavior in asphalt binder. To evaluate the required amount of thermal energy to overcome the intermolecular force was calculated using the Arrhenius model based on viscosity data captured from viscosity test. The rotational viscosity is determined by measuring the required torque to maintain the cylindrical spindle rotational speed while submerging in a steel mold filled with binder at a constant rate (AASHTO TP4). It is wise to note that viscosity value is

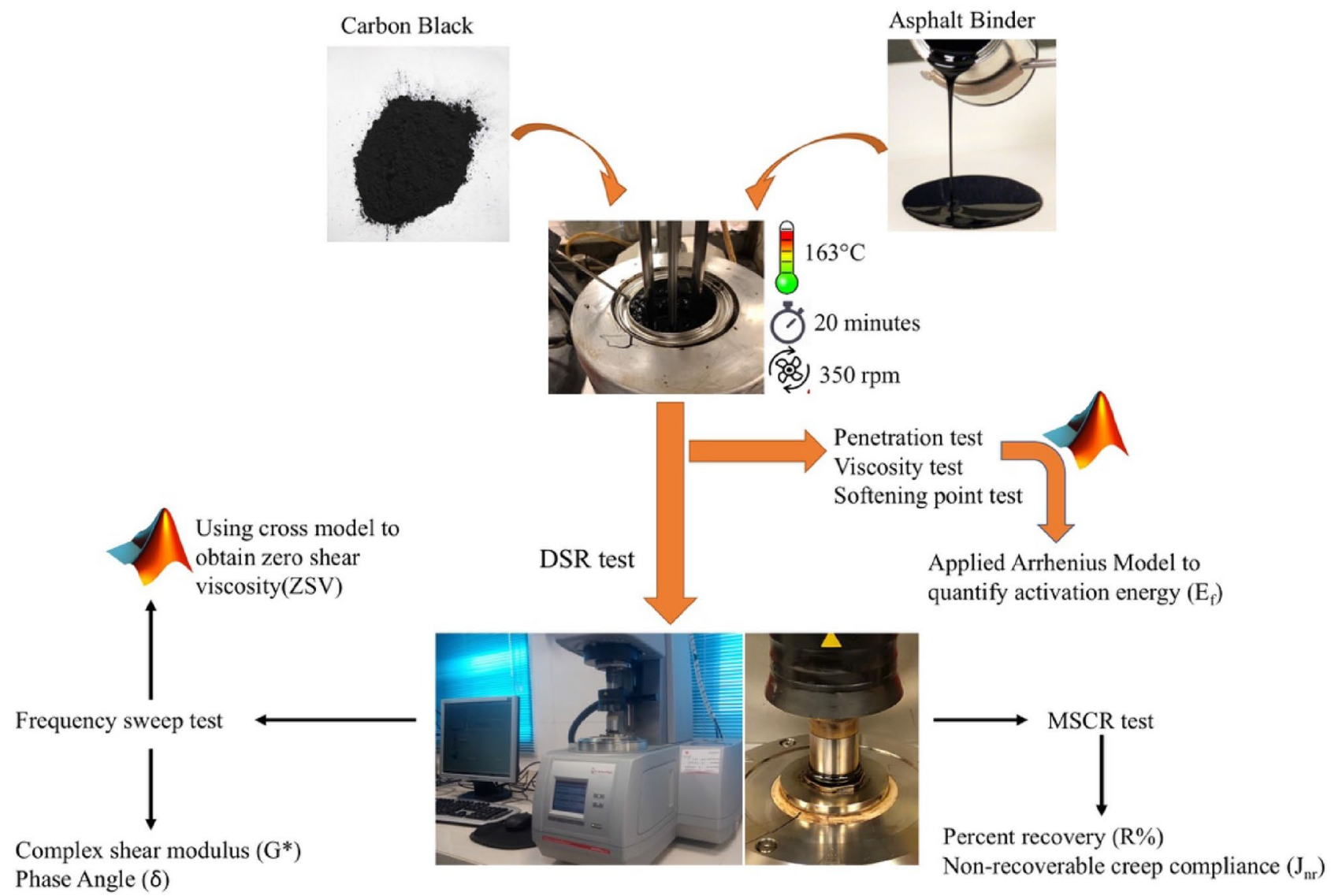

Fig. 1 A graphical schematic of modification and testing process 
measured at constant speed of 20 RPM. Moreover, it was decided to measure the viscosity of virgin and CB-modified asphalt binders at $135,150,165^{\circ} \mathrm{C}$.

\subsubsection{Superpave specification parameter}

Bahia and Anderson [46] and Robert et al. [47] have reported that complex shear modulus $\left(G^{*}\right)$ and phase angle $(\delta)$ obtained from DSR determine the relationship between asphalt binder stiffness and its deformation type. It is justifiable to regard rutting resistance at high service temperatures in the early age of pavement serviceability, because, at these temperatures, the behavior of asphalt binder is more viscous rather than elastic. The higher the $G^{*}$, and lower the $\delta$, the greater the rutting resistance of asphalt binder. An asphalt binder with higher value of $G^{*}$ is stiffer and provides increased resistance against deformation. Therefore, SHRP proposed to use $G^{*} / \sin \delta$ as the Superpave rutting specification parameter. It is wise to note that after emerging polymer-modified asphalt binders, the multi stress creep and recovery test has been introduced to describe the stress dependency and anti-rutting resistance of modified asphalt binders.

To provide robust reasoning about the influence of $C B$ on the high-temperature viscoelastic behavior of binder, the Superpave parameter $\left(G^{*} / \sin \delta\right)$ was measured for both virgin and modified specimens at various test temperatures. Moreover, to observe the interaction of temperature and the CB-dosage effects on the Superpave high-temperature parameter, it was decided to define a rutting resistance improvement ratio for the modified binder which was obtained by dividing $G^{*} / \sin \delta$ of CB-modified binder to that of the control sample (i.e., virgin binder). To this end, the result of the frequency sweep test at $10 \mathrm{rad} / \mathrm{s}$ was selected to calculate this ratio.

\subsubsection{Zero shear viscosity}

At high temperatures, virgin asphalt binder behaves like a Newtonian fluid making asphalt mixtures more prone to rutting. When the rate of loading is very low, the molecular transition rate of asphalt binder is very slow that makes its behavior like Newtonian fluids, so acclimation microstructure is done uniformly, on the other word; the asphalt binder, in this case, is in equilibrium condition. In this condition, the viscosity of binder is called zero shear viscosity (ZSV). However, asphalt binder demonstrates pseudoplasticity, or shear-thinning fluid behavior, due to which binder's viscosity decreases with an increase in shear rate. At very low shear rates, the pseudoplastic liquids act like Newtonian fluids, and their viscosity is not dependent on shear rate or loading frequency. This viscosity, i.e., $\eta_{0}$, is referred to as zero shear viscosity (ZSV) [48]. Sybilski [49] illustrated that ZSV can be used for evaluating the rutting resistance of asphalt mixture. There are several techniques to compute the ZSV value for asphalt binders. In this study, the frequency sweep test was used to compute the ZSVassuming that the asphalt binder is a cross fluid. A cross fluid is a Newtonian liquid whose viscosity depends on the applied shear rate [49-51]. As a result, the flow curves were fitted to the cross model (Eq. 1).

$\frac{\eta^{*}-\eta_{\infty}^{*}}{\eta_{0}^{*}-\eta_{\infty}^{*}}=\frac{1}{1+(k \omega)^{m}}$

where $\eta^{*}$ is complex viscosity, $\eta_{0}^{*}$ is zero shear viscosity (ZSV), $\eta_{\infty}^{*}$ is limiting viscosity, $\omega$ is angular frequency $(\mathrm{rad} / \mathrm{s})$, and $\mathrm{m}$ and $\mathrm{k}$ are constant coefficients. The frequency sweep test is commonly implemented between 0.1 and $100 \mathrm{rad} / \mathrm{s}$. Within this range, it is assumed that $\eta^{*} \gg n_{\infty}^{*}$. As a result, the cross model can be rewritten as (Eq. 2):

$\eta^{*}=\frac{\eta_{0}^{*}}{1+(k \omega)^{m}}$

Using the curve-fitting tool in Matlab ${ }^{\circ}$, it would be possible to fit Eq. (2) to complex viscosity versus angular frequency data point for each temperature to determine the ZSV value. The ZSV value is straightly proportional to permanent deformation resistance, and a greater ZSV is representative of higher anti-rutting resistance in asphalt binder [14]. To capture the viscoelastic properties of virgin and modified asphalt binders, the frequency sweep test was performed under a controlled-strain mode at frequencies ranging from 0.1 to $100 \mathrm{rad} / \mathrm{s}$. The shear strain of $1 \%$ was selected to ensure that the asphalt binder response remains in the linear viscoelastic zone. Also, to capture the rutting behavior of asphalt binders, the frequency sweep test was conducted at temperatures above $46^{\circ} \mathrm{C}-46,52$, 58 , and $64^{\circ} \mathrm{C}$-which the rutting is most likely to happen.

\subsubsection{Average recovery percentage and non-recoverable creep compliance}

Multi stress creep and recovery (MSCR) test has been alternatively used for examining the rutting resistance of asphalt binders when Superpave specification parameter fails to determine a justifiable correlation between loading type, loading level, and stress level. Average recovery percentage $(R \%)$, which is calculated based on the data acquired from MSCR test, can directly examine the elastic response of asphalt binders under creep and recovery loading [52]. The non-recoverable creep compliance $\left(J_{n r}\right)$ that is also obtained from the MSCR test is a useful parameter to examine the rutting behavior of asphalt binder. In this study, the multi stress creep and recovery (MSCR) test 
was conducted following the ASTM D7405-15 standard. The loading and recovery durations, respectively, were 1 and $9 \mathrm{~s}$. Ten creep-recovery cycles were applied for each stress levels: 0.1 and $3.2 \mathrm{kPa}$. It should be noted that the frequency sweep and MSCR tests were performed by an Anton Paar rheometer A101 with 25-mm diameter parallel plates spaced with a 1-mm gap opening at 46, 52, 58, and $64^{\circ} \mathrm{C}$, to capture viscoelastic properties of virgin and CB-modified asphalt binders.

\subsubsection{Activation energy investigation method}

Since asphalt binder is a viscoelastic material, its viscous portion can be considered as a thermally activated process in which the molecules of binder should overcome an energy barrier to move. In other words, when asphalt binder flows, the layer of molecules slide on each other, and as a result, intermolecular forces cause resistance against flow [49]. The amount of energy required to overcome this resistance called activation energy, which can be obtained by employing the Arrhenius model as follows:

$\eta=A e^{\frac{E_{f}}{R T}}$

where $\eta$ is the viscosity of asphalt binder, $T$ is the temperature in Kelvin, $A$ is a model constant, $E_{\mathrm{f}}$ is the activation energy, and finally, $R$ is the universal gas constant equal to $8.314 \mathrm{~J} \mathrm{~mol}^{-1} \mathrm{~K}^{-1}$.

To simplify this equation for obtaining $E_{f}$ Eq. (3) can be rewritten as follows:

$\ln \eta=+\ln A \frac{E_{f}}{R T}$

Equation 4 shows that the slope of $\ln \eta$ versus $1 / T$ equals to $E_{\mathrm{f}} / R$, because the trend is linear. Therefore, it is possible to determine the activation energy by considering the slop of In $\eta$ versus $1 / T$. The slope of this plot gives us the $E_{f} / R$. As a result, considering the universal gas constant, $E_{\mathrm{f}}$ can be calculated easily. For instance, the trend for obtaining the $E_{\mathrm{f}}$ value for the virgin binder is shown in Fig. 2.

\section{Result and discussion}

\subsection{Physical properties}

According to Table 2, increasing CB content decreases the penetration of asphalt binder, making it stiffer, and the lowest penetration value is obtained at the CB of $15 \%$. Such behavior can be attributed to the consistency of the modified asphalt binder due to the interaction of $C B$ particles with the asphalt binder components. Generally, increasing $C B$ content in the modified blend increases the

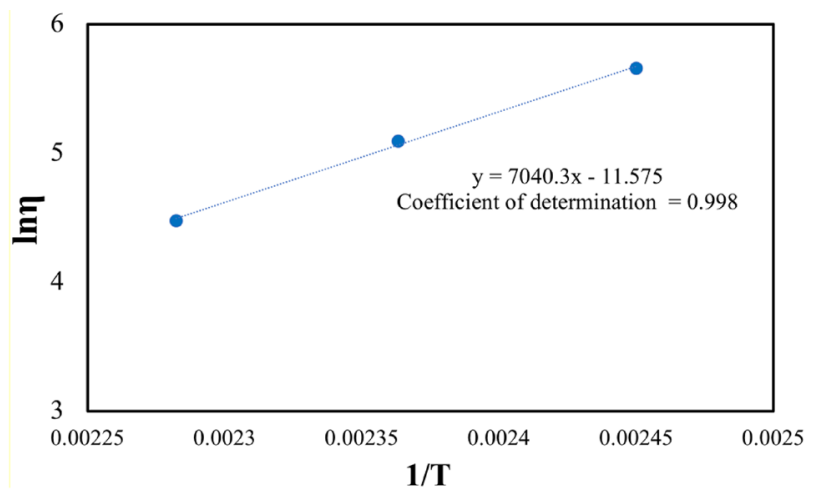

Fig. 2 Ln versus $1 / T$ for virgin asphalt binder

Table 2 Physical properties of virgin and CB-modified asphalt binders

\begin{tabular}{llll}
\hline Sample & $\begin{array}{l}\text { Viscosity }\left(135^{\circ} \mathrm{C}\right) \\
\text { pa s }\end{array}$ & $\begin{array}{l}\text { Penetration }\left(25^{\circ} \mathrm{C}\right) \\
0.1 \mathrm{~mm}\end{array}$ & $\begin{array}{l}\text { Softening } \\
\text { point }\left({ }^{\circ} \mathrm{C}\right)\end{array}$ \\
\hline OB & 0.287 & 98 & 45 \\
CB 5\% & 0.350 & 81 & 47.3 \\
CB 10\% & 0.427 & 72 & 48.4 \\
CB 15\% & 0.436 & 69 & 48.6 \\
\hline
\end{tabular}

ratio of asphaltene to maltene, because the light portion of asphalt binder is absorbed by CB particles, which transform it high viscosity asphalt binder. The softening point, however, increases with the increase in $\mathrm{CB}$ content, which can be related to change in the ratio of maltene/asphaltene as well.

CB particles absorb the light portion of the binder, increasing the ratio of asphaltene/maltene, and as therefore, the fluidity (the ability of an asphalt binder to flow easily) of the binder decreases. Consistently, the results of the viscosity of virgin and modified asphalt binder illustrate a good agreement with binder fluidity as the great viscosity related to CB $15 \%$ (Table 2).

\subsection{Viscoelastic properties}

Figure 3 presents two main viscoelastic parameters for virgin and CB-modified asphalt binders. As can be seen from Fig. 3a, increasing $C B$ content in the blend increases the complex shear modulus in which the highest value attributed to CB 15\%. With an explorer in Fig. 3, there is clear trend of decreasing phase angle with adding $C B$ in which the lowest phase angle attributed to the CB 15\% that implies the idea that it has more rutting resistance compared to others. It can be related to the high surface area of CB particles that absorbs the aromatic and light 

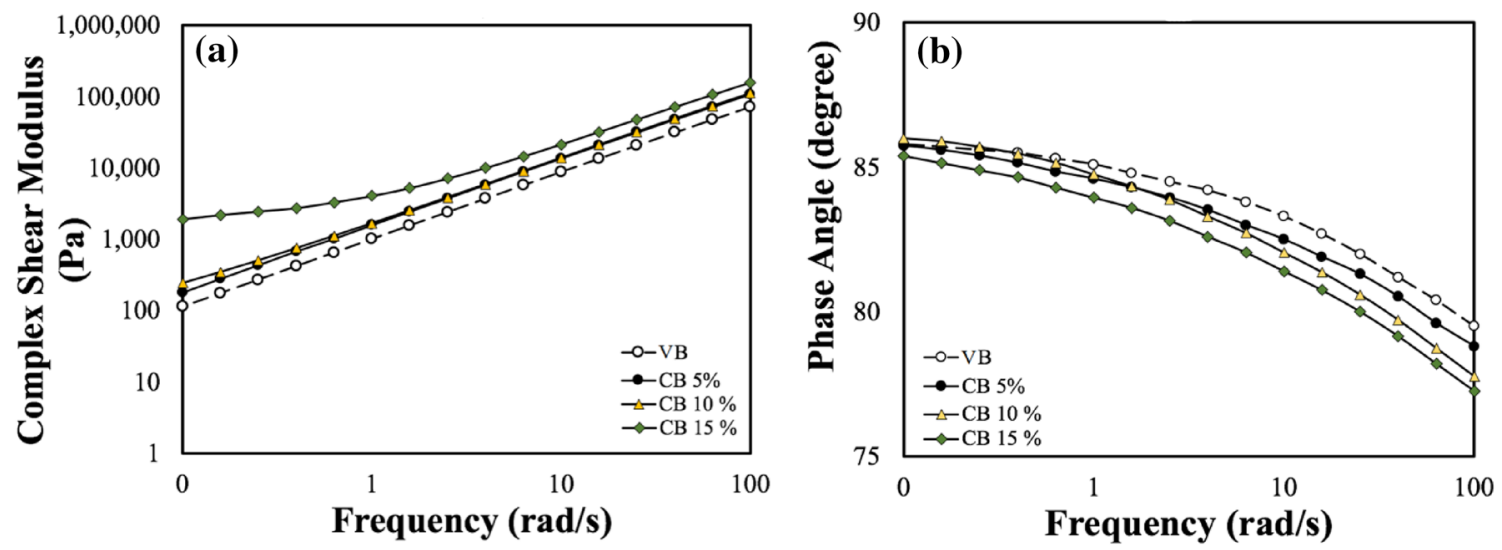

Fig. 3 a Complex shear modulus $\left(G^{*}\right)$ and phase angle $(\delta)$ versus loading frequency $(\omega)$ for virgin and CB-modified asphalt binders at $46{ }^{\circ} \mathrm{C}$

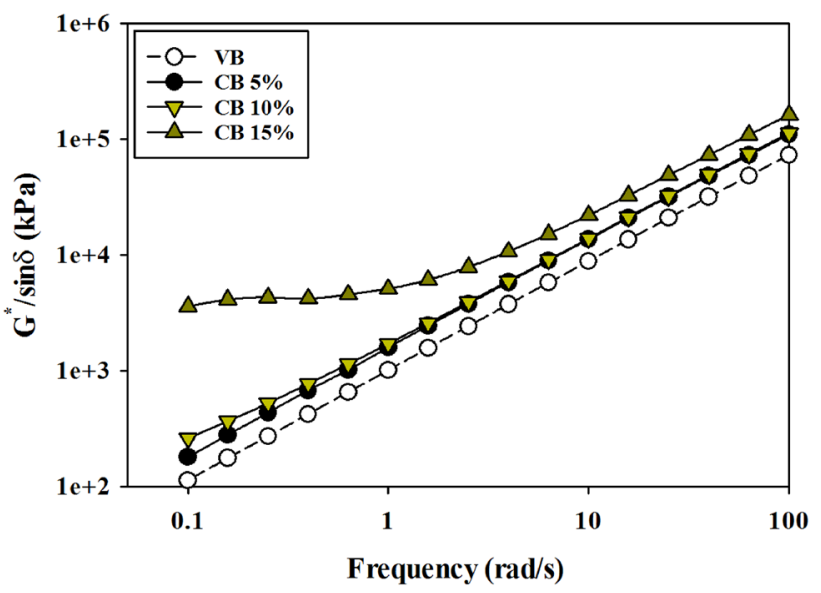

Fig. $4 G^{*} / \sin \delta$ for virgin and CB-modified binders at $46^{\circ} \mathrm{C}$

portion of the asphalt binder. As a result, the binder will be stiffer compared to the virgin asphalt binder.

Figures 4 and 5 present the Superpave rutting parameter for both virgin and CB-modified binders. It can be seen that modifying binder with $C B$ increases the value of $G^{*} / \sin \delta$. At low frequencies, i.e., the frequencies smaller than $3.98 \mathrm{rad} / \mathrm{sec}$, the difference between the value of the $G^{*} / \sin \delta$ for the virgin binder and the modified binders increases gradually with a decrease in frequency. It is worth noting that, at both temperatures, the difference between the $G^{*} / \sin \delta$ values is the largest when comparing virgin binder and the binder modified with $15 \% \mathrm{CB}$. Modification of asphalt binder with $C B$ increases the $G^{*}$ / $\sin \delta$ value, which leads to improved rutting resistance compared with the virgin asphalt binder.

\subsection{Superpave rutting specification parameter}

Comparing Figs. 4 and 5 proves the key role of temperature on influencing the rutting resistance of asphalt

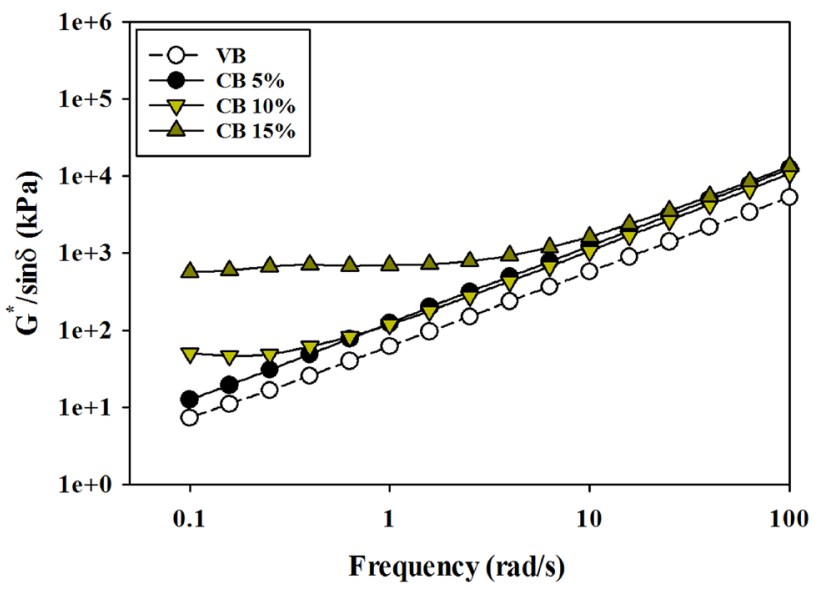

Fig. $5 G^{*} / \sin \delta$ for virgin and CB-modified binders at $64^{\circ} \mathrm{C}$

binder. At $64{ }^{\circ} \mathrm{C}$, the $G^{*} / \sin \delta$ values are smaller than the values calculated for binders tested at $46^{\circ} \mathrm{C}$. Also, raising the temperature to $64^{\circ} \mathrm{C}$ makes the influence of $\mathrm{CB}$ more distinguishable at lower frequencies due to the fact that at higher temperature or lower frequencies, CB-modified binder presents a high ratio of asphaltene to maltene enhancing binder temperature susceptibility, where the asphalt binder is the most prone to rutting.

Figure 6 presents the rutting improvement ratio based on data obtained from the frequency sweep test at $10 \mathrm{rad} / \mathrm{s}$. Whereby this ratio can be calculated by dividing $G^{*} / \sin \delta$ of CB-modified binder to $G^{*} / \sin \delta$ of the virgin binder at the loading frequency of $10 \mathrm{rad} / \mathrm{s}$. The rutting resistance ratio of the binder modified with $\mathrm{CB}$ increases with increase in temperature. This improvement can be justified by the increased ratio of asphaltene/maltene due to absorption of a portion of maltenes by CB particles. Moreover, when the specimens are subjected to temperature increases, the increased rate of $G^{*} / \sin \delta$ for asphalt binder modified with $10 \% \mathrm{CB}$ is greater than those 


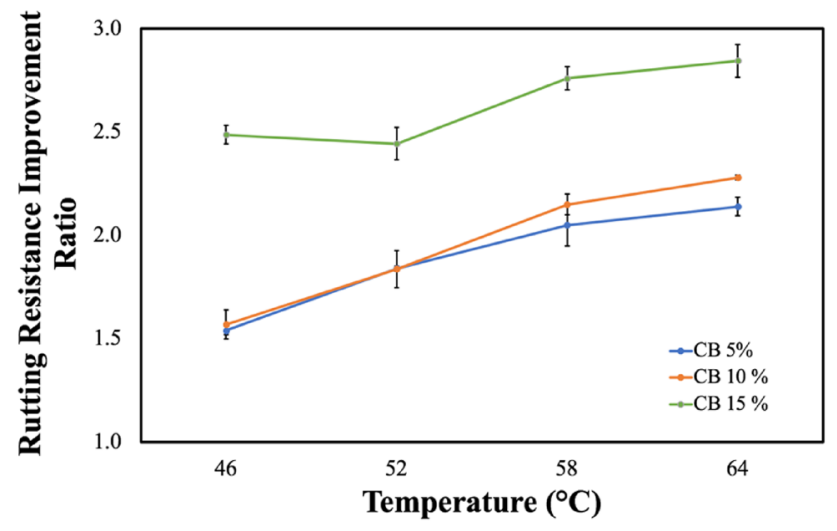

Fig. 6 Rutting resistant improvement ratio at all test temperatures at $10 \mathrm{rad} / \mathrm{s}$

of asphalt binders modified with $5 \%$ and $15 \%$ CB. In other words, the asphalt binders modified with $5 \%$ and $15 \%$ CB become less temperature susceptible, in the context of rutting, which can be attributed to the presence of very small amounts of light portion in these modified binders.

According to Fig. 6, by increasing CB content in the blend, the rutting resistance improvement ratio raises, essentially the highest improvement attributed to the $C B$ $15 \%$. It can be justified in terms of the interaction between CB particles and asphaltene in binder colloidal structure. Due to the high absorption percentage of $C B$ particles, modifying binder with $C B$ causes $C B$ particles covered with a layer of asphaltene leading the binder to low mobility. As a result, the viscosity of the modified asphalt binder is increased. To support it, the viscosity value given in Table 2 presents a good agreement to this explanation.

\subsection{Zero shear viscosity}

As it was mentioned in the test method section, the cross model is a suitable means for computing the ZSV value of asphalt binder. This model consists of three parameters; zero shear viscosity, complex viscosity, and two coefficients that can be obtained using the fit-curve technique performed on the data acquired from the frequency sweep test at each temperature. A nonlinear regression analysis was used in Matlab to fit Eq. (2) to the acquired data. Figure 7 represents the complex viscosity versus angular frequency data obtained from a frequency sweep test at the test temperature of $64^{\circ} \mathrm{C}$. As it can be observed CB 10\% and $15 \%$ have a high complex viscosity at low frequencies, because binder modification improves permanent deformation resistance.

To characterize the rutting performance of CB-modified binders at high temperatures, the ZSV values were calculated for both modified and virgin binders. Table 3 shows

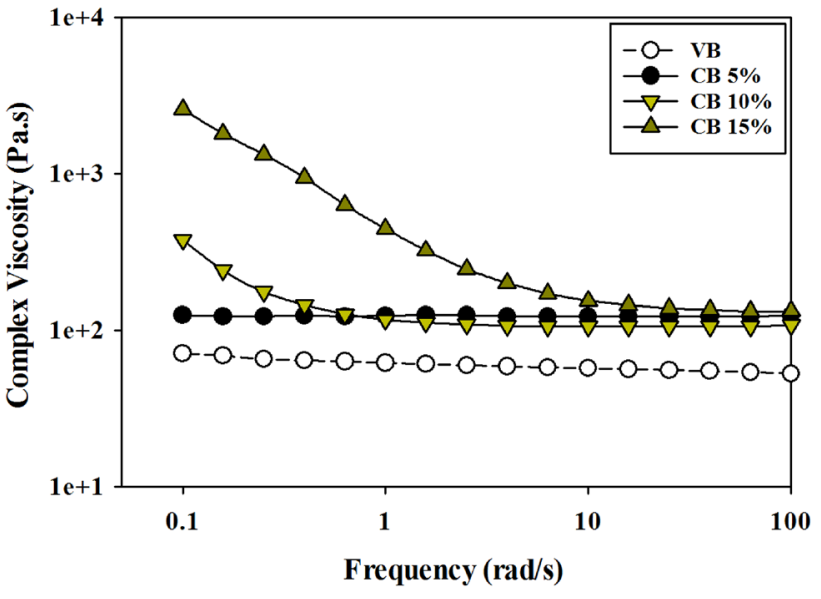

Fig. 7 Complex viscosity versus angular frequency at $64^{\circ} \mathrm{C}$

the computed ZSV for both virgin and CB-modified asphalt binder. With an explore in this table, it is obvious that increasing test temperature reduces ZSV value. Moreover, extending the $\mathrm{CB}$ content in the modified blend up to $10 \%$ by weight of total binder asphalt binder increases the ZSV value, and for further content, it is reduced.

To have a better understanding, the ZSV values calculated for modified binders were divided by that of virgin binder to obtain indices. These indices were used for making a comparison between the CB-modified binders in terms of rutting resistance enhancement. Note that in Fig. 8, increasing the amount of $C B$ in the blend increases the ZSV value. At all the temperatures, CB $10 \%$ has the highest indices, followed by CB $15 \%$ and then CB 5\%. The calculated index for $C B$ 5\% remains constant at all the test temperatures. CB $10 \%$ and CB $15 \%$ have the highest indices at the test temperature of $46^{\circ} \mathrm{C}$, and their indices, with a slight reduction at the test temperature of $52{ }^{\circ} \mathrm{C}$, remain virtually constant at higher tests temperatures. This stable behavior can be attributed to the fact that the binder softens and loses its stiffness at these high temperatures (i.e., $52{ }^{\circ} \mathrm{C} \leq T \leq 64^{\circ} \mathrm{C}$ ). It is worth highlighting that the results obtained from the analysis performed on ZSV values are in contradiction with those obtained from the analysis

Table 3 Value for virgin and CB-modified asphalt binders at different temperatures

\begin{tabular}{lrrrr}
\hline \multicolumn{4}{l}{ Zero shear viscosity $(\mathrm{Pa} \mathrm{s})$} \\
\hline Temperature $\left({ }^{\circ} \mathrm{C}\right)$ & \multicolumn{1}{l}{46} & \multicolumn{1}{l}{5} \\
\hline Virgin binder & 8880 & 3440 & 1380 & 573 \\
CB 5\% & 9217 & 3563 & 1495 & 642 \\
CB 10\% & 32,057 & 10,010 & 3077 & 1203 \\
CB 15\% & 31,790 & 8744 & 2305 & 923 \\
\hline
\end{tabular}




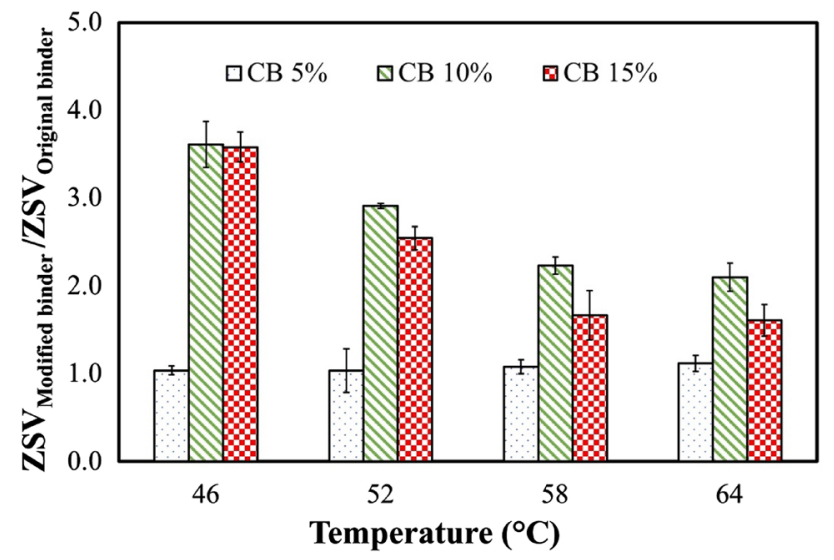

Fig. 8 Rutting resistant improvement at all test temperature based on ZSV

performed on Superpave high-temperature specification parameter.

\subsection{Average recovery percentage and non-recoverable creep compliance}

The average recovery percent (R\%) obtained from the MSCR test is representative of asphalt binder's elasticity under cyclic loading, and the higher the $R \%$, the higher the deformation recovery. As a result, it can be stated that a binder with a higher $R \%$ has more elasticity. Figure 9 shows the recovery percentage of the virgin binder and CB-modified asphalt binder at two stress levels: 0.1 and $3.2 \mathrm{kPa}$, respectively. The highest $R \%$ is obtained from the binder modified with $10 \% \mathrm{CB}$. Therefore, the addition of CB at content about $10 \%$ of binder weight results in enhancement of elastic recovery as well as rutting resistance in asphalt binder. Moreover, increasing the $C B$ to a percentage beyond $10 \%$ (e.g., $15 \%$ ) results in a reduction in elastic recovery. At all the selected test temperatures, both virgin and CB-modified asphalt binders show sensitivity to change in stress level, i.e., the $R \%$ values calculated for all the binder types are greater at the low-stress level of $0.1 \mathrm{kPa}$ than those calculated for the stress level of $3.2 \mathrm{kPa}$. The reason for the reduction in $R \%$ at higher stress levels can be attributed to the stress dependency of asphalt binder in which higher stress level making the viscous behavior more dominant. Also, increasing the temperature causes reduction in the recovery percentage of asphalt binder which can be attributed to increasing of maltene in asphalt binder at higher temperatures making the viscous behavior more dominant [50]. At the stress level of $0.1 \mathrm{kPa}$, CB 10\% and CB15\% show a better performance in terms of having higher $R \%$ values at all the test temperatures in comparison with virgin asphalt binder. Such improvement may be attributed to the reduction in maltene absorbed by $\mathrm{CB}$ particles making the asphaltenes' role more dominant. Moreover, the highest recovery percentage is related to the modified asphalt binder containing $10 \% \mathrm{CB}$.

The non-recoverable creep compliance $\left(J_{n r}\right)$ is also obtained from the MSCR test and is used for characterizing the viscoelastic response of asphalt binder. The lower the $J_{\mathrm{nr}}$ value, the higher the rutting resistance. According to the findings shown in Fig. 10, the presence of CB results in the reduction in $J_{n r}$ value at both stress levels, and this reduction is the greatest at the test temperature of $64^{\circ} \mathrm{C}$, followed by $58^{\circ} \mathrm{C}$. Moreover, at the high-stress level of $3.2 \mathrm{kPa}$, the influence of $\mathrm{CB}$ on the viscoelastic response is greater than the low-stress level of $0.1 \mathrm{kPa}$. Note that in Fig. 10, at the test temperature of $64^{\circ} \mathrm{C}$, the $J_{\mathrm{nr}}$ value of asphalt binder modified with $10 \%$ CB has decreased for
Fig. 9 Recovery percentage of virgin and CB-modified asphalt binders

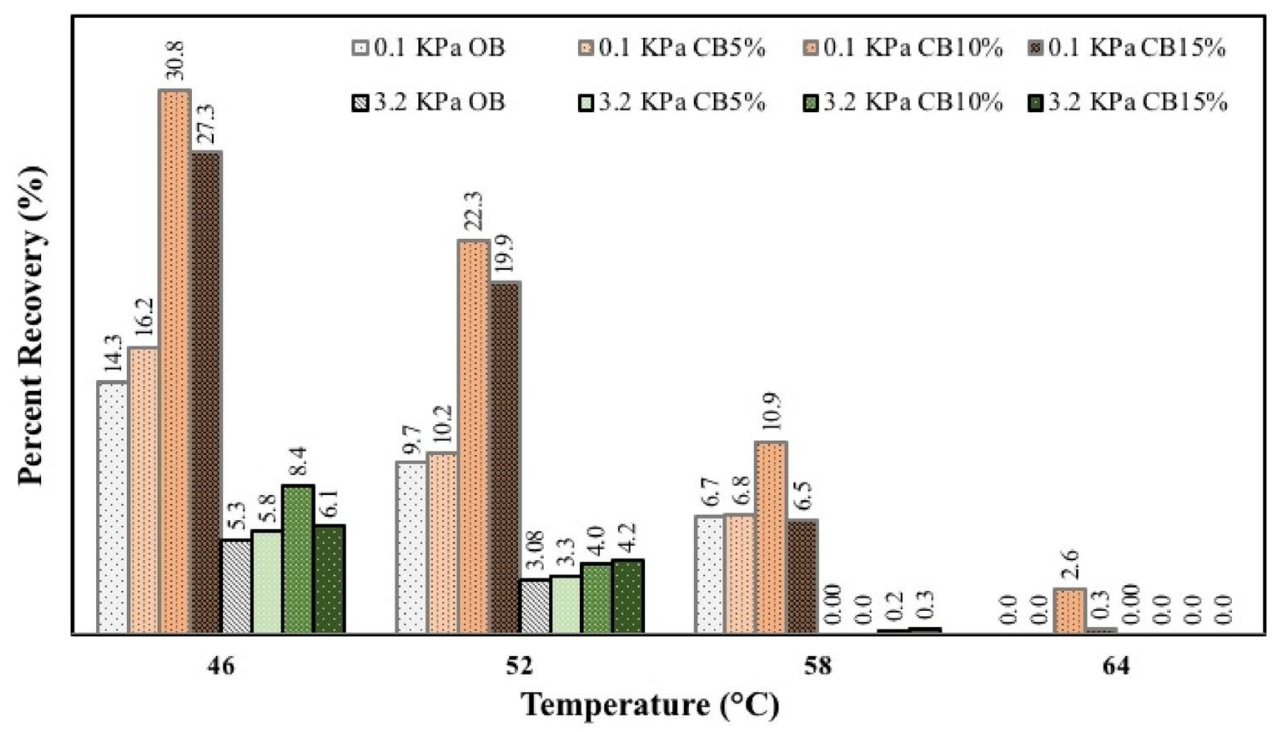


Fig. 10 Non-recoverable creep compliances of virgin and CBmodified asphalt binders

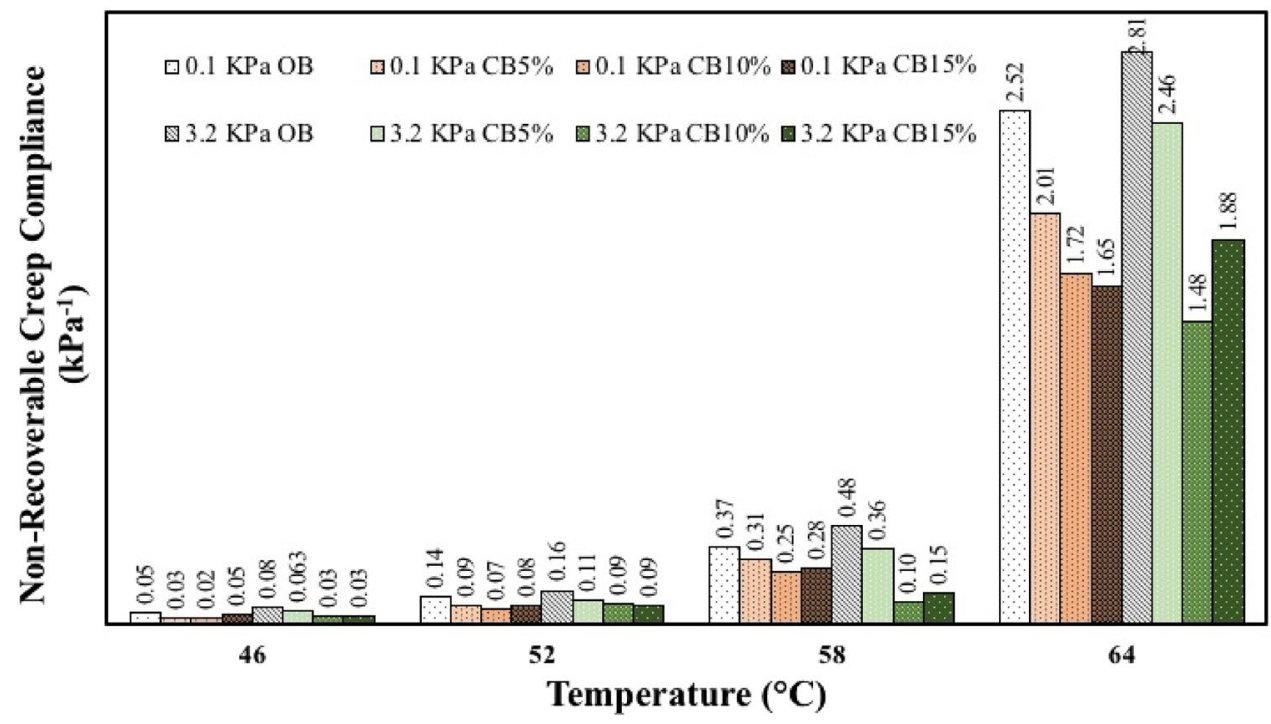

about $32 \%$ and $48 \%$, respectively, at the stress levels of $0.1 \mathrm{kPa}$ and $3.2 \mathrm{kPa}$.

After considering all analysis approaches, it can be concluded that the ZSV analysis can be more representative of high-temperature performance compared with the Superpave rutting specification parameter when comparing those to the MSCR result analysis. In other words, according to Superpave rutting specification, for a high dosage of CB (i.e., 15\%), the rutting resistance was increased, while, according to MSCR test result, the elastic recovery of asphalt binder decreased. This discrepancy can be explained in part by the idea that the presence of high content of $C B$ in the modified blend increases the probability of phase segregation in the asphalt binder. As a result, deposition of heavy molecules might take place, which can disturb the stability of maltene and asphaltene phases in the CB-modified asphalt binder. Moreover, the ZSV analysis placed in a good agreement with the MSCR test result. Simply put, it can be stated that ZSV can be used as an excellent rutting resistance representative approach. Based on such result and discussion, it can be concluded that the CB content of $10 \%$ is the maximum recommended value for increasing the resistance of asphalt binder against rutting.

\subsection{Activation energy}

Figure 11 presents the activation energy that requires to overcome the barrier force between modified asphalt binder's molecules to move. This feature is significant from energy consumption at the hot mix asphalt (HMA) production process, in which, higher activation energy implies the idea that the molecules of binder need more kinematic energy to overcome the intermolecular force to the fluid. As can be seen from Fig. 11, modifying asphalt binder

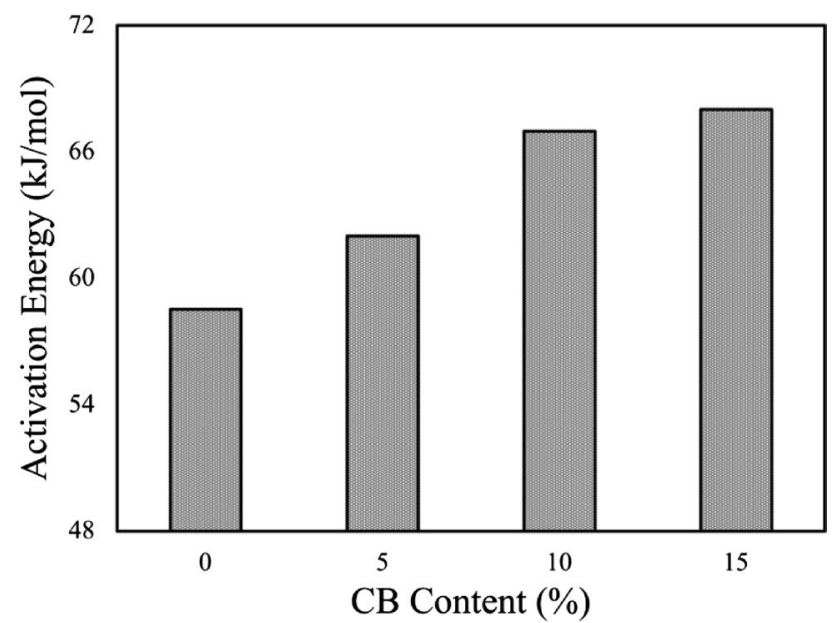

Fig. 11 Required activation energy for virgin and CB-modified asphalt binders

with $C B$ increases activation energy as the highest value related to modified asphalt binder containing $15 \%$ of $C B$. It implies the fact that CB interacts with asphalt binder maltene portion that results in larger hydrocarbon chains. Since the high activation energy implies the fact that in the HMA production process, more heat energy required to make the binder fluid enough for pumping and mixing with aggregate. As a result, it increases the energy consumption and then increases the final cost of the material. Moreover, it increases greenhouse gas emission, especially in the production process of HMA.

It was claimed that the extra thermal energy generated for modified asphalt binders, make asphalt binder more susceptible to short-term aging [53]. From this standpoint, it is recommended to evaluate the short-term aging of CBmodified asphalt binder for future works. 


\section{Conclusion}

This study set out to evaluate the effect of $C B$ on asphalt binder rheological performance and fluid resistance at high service temperatures. To capture such objectives, three approaches were adopted to assess CB on rutting and rheological performance of asphalt binders: Superpave rutting specification parameter $\left(G^{*} / \sin \delta\right)$, zero shear viscosity (ZSV), and multiple stress creep and recovery (MSCR) test as well as a cross model were used to quantify of fluid resistance by introducing activation energy concept. This study led to the following outcomes:

- Increasing $\mathrm{CB}$ content in the modified asphalt binder up to $10 \%$ amplifies the enhancement trend of asphalt binder's rutting resistance.

- CB-modified asphalt binder indicates low non-recoverable creep compliance compared to the virgin asphalt binder, especially for $10 \%$ CB content.

- Extending carbon black content in the modified asphalt binder results in stiffer asphalt binder in which the highest stiffer asphalt binder containing $15 \%$ CB content.

- Addition of $C B$ beyond $10 \%$ either maintains or degrades the elastic recovery and non-recoverable creep compliance.

- The effect of CB on the elastic properties of asphalt binder at the high-stress level is more representative than lower loading stress level.

- Binder modified with $10 \%$ CB performs better in terms of ZSV and MSCR values on $J_{n r}$ either in 0.1 or $3.2 \mathrm{kPa}$ stress level.

- Comparing different rutting assessment approaches shows that the zero shear viscosity (ZSV) can be used as an excellent representative approach for evaluating the elastic behavior of asphalt binder for high service temperature performance.

- CB-modified asphalt binders indicated higher activation energy in comparison with the virgin binder that increases the total material cost of HMA during the plant production process.

This study focused on the effect of $C B$ on rutting resistance of asphalt binder in which $C B$ content of 10 percent was concluded as the optimum percentage that valid for high-temperature performance based on the analysis discussed in this study. However, for determining the optimum content of $C B$, it is recommended to evaluate the low and intermediate temperature performance of CB-modified asphalt binder. Since there is no comprehensive state of the art on the performance of
CB-modified asphalt binder and mixture, it is recommended to investigate the physicochemical properties of CB-modified asphalt binder in terms of chemical functional groups (i.e., carbonyl and sulphoxide functional groups) and the short/long term aging resistance of CBmodified asphalt binder. Moreover, it is suggested to extend the analysis level from asphalt binder to asphalt mixture and then validate the ZSV rutting evaluation approach with rutting performance of asphalt mixture to establish a promising correlation between them.

Acknowledgements The authors would like to thank the assistance of Mr. Nader Goudarzi for kind help through the modification process of CB-asphalt binders.

Conflict of interest The authors declare that there is no any conflict of interest regarding this study.

\section{References}

1. Shell Bitumen (1995) The shell bitumen industrial handbook. Thomas Telford Ltd, London

2. Fini EH, Hajikarimi P, Rahi M, Moghadas Nejad F (2015) Physiochemical, rheological, and oxidative aging characteristics of asphalt binder in the presence of mesoporous silica nanoparticles. J Mater Civ Eng 28(2):04015133

3. Nakhaei M, Naderi K, Nasrekani AA, Timm DH (2018) Moisture resistance study on PE-wax and EBS-wax modified warm mix asphalt using chemical and mechanical procedures. Constr Build Mater 189:882-889

4. Notani MA, Moghadas Nejad F, Khodaii A, Hajikarimi P (2019) Evaluating fatigue resistance of toner-modified asphalt binders using the linear amplitude sweep test. Road Mater Pavement Des 20(8):1927-1940

5. Aflaki S, Hajikarimi P, Fini EH, Zada B (2014) Comparing effects of biobinder with other asphalt modifiers on low-temperature characteristics of asphalt. J Mater Civ Eng 26(3):429-439

6. Rahbar-Rastegar R, Daniel JS, Dave EV (2018) Evaluation of viscoelastic and fracture properties of asphalt mixtures with long-term laboratory conditioning. Transp Res Rec 2672(28):503-513

7. Zaniewski JP, Pumphrey ME (2004) Evaluation of performance graded asphalt binder equipment and testing protocol. Asphalt technology program, 107

8. White $G$ (2019) Evaluation of a non-nuclear density gauge as an alternate to destructive coring for airport asphalt acceptance testing. SN Appl Sci 1(8):921

9. Daghighi AMIN, Nahvi A (2014). Effect of different additives on fatigue behaviour of asphalt mixtures. In: Construction materials and structures: proceedings of first international conference on construction materials and structures. IOS Press Amsterdam, pp 601-607

10. Arabzadeh A, Guler M (2019) Thermal fatigue behavior of asphalt concrete: a laboratory-based investigation approach. Int J Fatigue 121:229-236

11. Kok BV, Kuloglu N (2007) The effects of different binders on mechanical properties of hot mix asphalt. Int J Sci Technol 2(1):41-48

12. Notani MA, Hajikarimi P, Nejad FM, Khodaii A (2020) Rutting resistance of toner-modified asphalt binder and mixture. 
Int J Pavement Res Technol. https://doi.org/10.1007/s4294 7-019-0131-z

13. Golalipour A (2011) Modification of multiple stress creep and recovery test procedure and usage in specification (Doctoral dissertation)

14. Rahi M, Fini EH, Hajikarimi P, Nejad FM (2014) Rutting characteristics of styrene-ethylene/propylene-styrene polymer modified asphalt. J Mater Civ Eng 27(4):04014154

15. Daniel JS, Corrigan M, Jacques C, Nemati R, Dave EV, Congalton A (2019) Comparison of asphalt mixture specimen fabrication methods and binder tests for cracking evaluation of field mixtures. Road Mater Pavement Des 20(5):1059-1075

16. Garba R (2002) Permanent deformation properties of asphalt mixtures. In: NVF Conference, vol 28

17. Sousa JB, Craus J, Monismith CL (1991) Summary report on permanent deformation in asphalt concrete (No. SHRP-A-318)

18. Centeno M, Sandoval I, Cremades I, Alarcon J (2008) Assessing rutting susceptibility of five different modified asphalts in bituminous mixtures using rheology and wheel tracking test (No. 08-0705)

19. Eisenmann J, Hilmer A (1987) Influence of wheel load and inflation pressure on the rutting effect at asphalt-pavements: experiments and theoretical investigations. In: International conference on the structural design

20. Hajikarimi P, Fakhari Tehrani F, Moghadas Nejad F, Absi J, Rahi M, Khodaii A, Petit C (2018) Generalized fractional viscoelastic modeling of low temperature characteristics of asphalt binders modified with polyphosphoric acid and distillate aromatic extracts oil. J Mater Civ Eng 30(7):04018147

21. Raj A, Krishnan JM (2019) Influence of gauge length on the measurement of resilient modulus of bituminous mixtures. SN Appl Sci 1(1):118

22. Polacco G, Berlincioni S, Biondi D, Stastna J, Zanzotto L (2005) Asphalt modification with different polyethylene-based polymers. Eur Polymer J 41(12):2831-2844

23. Notani MA, Moghadas Nejad F, Fini EH, Hajikarimi P (2019) Low-temperature performance of toner-modified asphalt binder. J Transp Eng Part B Pavements 145(3):04019022

24. Kim H, Lee SJ, Amirkhanian SN (2011) Rheology of warm mix asphalt binders with aged binders. Constr Build Mater 25(1):183-189

25. Mahmoudzadeh A, Golroo A, Jahanshahi MR, Firoozi Yeganeh $S$ (2019) Estimating pavement roughness by fusing color and depth data obtained from an inexpensive RGB-D sensor. Sensors 19(7):1655

26. Long CM, Nascarella MA, Valberg PA (2013) Carbon black vs. black carbon and other airborne materials containing elemental carbon: physical and chemical distinctions. Environ Pollut 181:271-286

27. Le Guern M, Chailleux E, Farcas F, Dreessen S, Mabille I (2010) Physico-chemical analysis of five hard bitumens: identification of chemical species and molecular organization before and after artificial aging. Fuel 89(11):3330-3339

28. Mohammadafzali M, Ali H, Sholar GA, Rilko WA, Baqersad M (2018) Effects of rejuvenation and aging on binder homogeneity of recycled asphalt mixtures. J Transp Eng Part B Pavements 145(1):04018066

29. Wang H, Lu G, Feng S, Wen X, Yang J (2019) Characterization of bitumen modified with pyrolytic carbon black from scrap tires. Sustainability 11(6):1631

30. Lamontagne J, Dumas P, Mouillet V, Kister J (2001) Comparison by Fourier transform infrared (FTIR) spectroscopy of different ageing techniques: application to road bitumens. Fuel $80(4): 483-488$
31. Sanada K, Tada Y, Shindo Y (2009) Thermal conductivity of polymer composites with close-packed structure of nano and micro fillers. Compos A Appl Sci Manuf 40(6-7):724-730

32. Chaala A, Roy C, Ait-Kadi A (1996) Rheological properties of bitumen modified with pyrolytic carbon black. Fuel 75(13):1575-1583

33. Alsalihi M, Hosseini A, Faheem A (2017) Direct characterization of aging diffusion in asphalt mixtures using micro-indentation and relaxation (MIR). In: Airfield and highway pavements 2017, pp 129-140

34. Shishehbor M, Pouranian MR, Ramezani MG (2019) Molecular investigations on the interactions of graphene, crude oil fractions and mineral aggregates at low, medium and high temperatures. Pet Sci Technol 37(7):804-811

35. López-Moro FJ, Moro MC, Hernández-Olivares F, WitoszekSchultz B, Alonso-Fernández M (2013) Microscopic analysis of the interaction between crumb rubber and bitumen in asphalt mixtures using the dry process. Constr Build Mater 48:691-699

36. Li C, Fan Z, Wu S, Li Y, Gan Y, Zhang A (2018) Effect of carbon black nanoparticles from the pyrolysis of discarded tires on the performance of asphalt and its mixtures. Appl Sci 8(4):624

37. Sasaki I (2003) Aging and rheological properties of carbon black modified asphalt binders. Publication of: ARRB Transport Research, Limited

38. Cong P, Xu P, Chen S (2014) Effects of carbon black on the anti aging, rheological and conductive properties of SBS/asphalt/ carbon black composites. Constr Build Mater 52:306-313

39. Divya PS, Krishnan JM (2019) How to consistently collect rheological data for bitumen in a Dynamic Shear Rheometer? SN Appl Sci 1(1):100

40. Desmazes C, Lecomte M, Lesueur D, Phillips M (2000) A protocol for reliable measurement of zero-shear-viscosity in order to evaluate the anti-rutting performance of binders. In: Proceedings of the papers submitted for review at 2nd Eurasphalt and Eurobitume congress, Held 20-22 Sept 2000, SPIN. Book 1- Session 1

41. Hajikarimi P, Rahi M, Moghadas Nejad F (2015) Comparing different rutting specification parameters using high temperature characteristics of rubber-modified asphalt binders. Road Mater Pavement Des 16(4):751-766

42. Rowe GM, D'Angelo JA, Sharrock MJ (2002) Use of the zero shear viscosity as a parameter for the high temperature binder specification parameter. In: 2nd International symposium on binder rheology and pavement performance

43. Pouranian MR, Imaninasab R, Shishehbor M (2018) The effect of temperature and stress level on the rutting performance of modified stone matrix asphalt. Road Mater Pavement Des 1-13

44. Bird RB, Armstrong RC, Hassager O (1987) Dynamics of polymeric liquids, vol 1: fluid mechanics

45. Anderson DA, Christensen DW, Bahia HU, Dongre R, Sharma MG, Antle CE, Button J (1994) Binder characterization and evaluation, volume 3: physical characterization. Strategic Highway Research Program, National Research Council, Report No. SHRP-A-369

46. Bahia HU, Anderson DA (1995) Strategic highway research program binder rheological parameters: background and comparison with conventional properties. In: Transportation research record (1488)

47. Roberts FL, Kandhal PS, Brown ER, Lee DY, Kennedy TW (1991) Hot mix asphalt materials, mixture design and construction. National Asphalt Pavement Association Research and Education Foundation, Lanham, MD

48. Yildirim Y, Solaimanian M, Kennedy TW (2000) Mixing and compaction temperatures for hot mix asphalt concrete (No. Report No. 1250-5). University of Texas at Austin. Center for Transportation Research 
49. Sybilski D (1996) Zero-shear viscosity of bituminous binder and its relation to bituminous mixture's rutting resistance. Transp Res Rec 1535(1):15-21

50. Sybilski D (1994) Relationship between absolute viscosity of polymer-modified bitumens and rutting resistance of pavement. Mater Struct 27(2):110-120

51. Fathi A, Haeri SM, Mazari M, Hosseini A, Kumar S, Zhu C (2019) Estimation of rocking capacity of soil-structure systems using a hybrid inverse solver. SN Appl Sci 1(7):703

52. Jamshidi A, Hamzah MO, Shahadan Z, Yahaya AS (2014) Evaluation of the rheological properties and activation energy of virgin and recovered asphalt binder blends. J Mater Civ Eng 27(3):04014135
53. Hofko B, Eberhardsteiner L, Füssl J, Grothe H, Handle F, Hospodka M, Grossegger D, Nahar SN, Schmets AJM, Scarpas A (2016) Impact of maltene and asphaltene fraction on mechanical behavior and microstructure of bitumen. Mater Struct 49(3):829-841

Publisher's Note Springer Nature remains neutral with regard to jurisdictional claims in published maps and institutional affiliations. 